Experiencias didácticas Juegos cooperativos-sensibilizadores para mejorar las actitudes hacia la discapacidad en educación física en educación primaria. Vol. 8, n. ${ }^{\circ}$ 1; p. 60-80, enero 2022. https://doi.org/10.17979/sportis.2022.8.1.8640

\title{
Juegos cooperativos-sensibilizadores para mejorar las actitudes hacia la discapacidad en educación física en educación primaria
}

\section{Cooperative-sensitizing games to improve attitudes towards disabilities in physical education in primary school}

\author{
Paula Mansilla y Jorge Abellán
}

Departamento de didáctica de la educación física, artística y música

Facultad de Educación de Cuenca

Universidad de Castilla-La Mancha

Contacto: Jorge.abellan@uclm.es

Cronograma editorial: Artículo recibido 27/07/2021 Aceptado: 06/11/2021 Publicado: 01/01/2022 https://doi.org/10.17979/sportis.2022.8.1.8640

Para citar este artículo utilice la siguiente referencia: Mansilla, P.; Abellán, J. (2022). Juegos cooperativos-sensibilizadores para mejorar las actitudes hacia la discapacidad en educación física en educación primaria. Sportis Sci J, 8 (1), 60-80. https://doi.org/10.17979/sportis.2022.8.1.8640

Contribución específica de los autores: Ambos autores han contribuido por igual a la elaboración de todo el manuscrito.

Financiación: No existió financiación para este proyecto.

Consentimiento informado participantes del estudio: No procede.

Conflicto de interés Los autores no señalan ningún conflicto de interés. 
Experiencias didácticas Juegos cooperativos-sensibilizadores para mejorar las actitudes hacia la discapacidad en educación física en educación primaria. Vol. 8, n. ${ }^{\text {1 }}$; p. 60-80, enero 2022.

https://doi.org/10.17979/sportis.2022.8.1.8640

\title{
Resumen
}

La aceptación del grupo de iguales es uno de los facilitadores para lograr la inclusión del alumnado con discapacidad y la Educación Física es una de las áreas más adecuadas para favorecer las actitudes positivas del grupo. Este trabajo presenta una propuesta didáctica de juegos cooperativos-sensibilizadores en Educación Física en la etapa de Educación Primaria. El objetivo principal de esta propuesta es analizar el efecto de la primera puesta en práctica de este tipo de juegos en las actitudes hacia la discapacidad de los participantes, además de analizar las barreras y facilitadores de su aplicación. Se desarrolla con 51 alumnos de quinto curso de Educación Primaria. Los resultados obtenidos muestran que la práctica de una serie de juegos cooperativos-sensibilizadores consigue que los participantes mejoren sus actitudes hacia la discapacidad en Educación Física. Entre los principales facilitadores destacan la valoración de capacidades y la empatía, mientras que el miedo del alumnado se ha identificado como la principal barrera. Se concluye que esta propuesta de juegos cooperativos-sensibilizadores puede resultar útil para sensibilizar hacia la discapacidad en Educación Física en Educación Primaria.

\section{Palabras clave}

Simulación; cooperación; juegos; actitudes; inclusión.

\begin{abstract}
The acceptance of peers is one of the facilitators to achieve the inclusion of students with disabilities and Physical Education is one of the most appropriate areas to promote positive attitudes. This work presents a didactic proposal of cooperative-sensitizing games in Physical Education in Primary Education. The main objective of this proposal is to analyse the effect of the first implementation of this type of games on the attitudes towards disability, and to analysing the barriers and facilitators of its implementation. It is developed with 51 students in the fifth year of Primary Education. The results obtained show that the practice of a series of cooperative-sensitizing games achieves that the participants improve their attitudes towards disability in Physical Education. Among the main facilitators, the assessment of capacities and empathy stand out, while the fear of the students has been identified as the main barrier. It is concluded that this proposal of cooperative-sensitizing games can be useful to sensitize towards disability in Physical Education in Primary Education.
\end{abstract}

\section{Keyword}

Simulation; cooperation; games; attitudes; inclusion. 
Experiencias didácticas Juegos cooperativos-sensibilizadores para mejorar las actitudes hacia la discapacidad en educación física en educación primaria. Vol. 8, n. 1; p. 60-80, enero 2022.

https://doi.org/10.17979/sportis.2022.8.1.8640

\section{Introducción}

El porcentaje total de alumnado con Necesidades Educativas Especiales integrado en centros ordinarios en el curso 2019-2020 es de un 83\% y atendiendo al tipo de discapacidad conocemos los siguientes datos: el 40,3\% corresponde a la categoría plurideficiencia, el $75,2 \%$ corresponde a discapacidad intelectual, el $83,3 \%$ corresponde a la categoría de trastorno general del desarrollo, el $88,3 \%$ corresponde a discapacidad motora, el 94,9\% corresponde a discapacidad auditiva, el 94,9\% a discapacidad visual y el 98,3\% corresponde a la categoría trastorno de la conducta/personalidad (Ministerio de Educación y Formación Profesional, 2021).

En este contexto, atender a la diversidad exige construir un modelo de escuela inclusiva en el que las prácticas estén basadas en los principios de tener en cuenta a todos los niños, independientemente de sus características y dificultades. Una escuela que promueva en sus aulas, en sus sesiones y con su alumnado, la democracia, la equidad, la igualdad, la pluralidad y la pertinencia, pero más aún que forme alumnos que reconozcan que lo más normal es que seamos diferentes (Simoni, Santillana y Yáñez, 2013).

Uno de los facilitadores de la inclusión del alumnado con discapacidad en el área de Educación Física es la actitud favorable de su grupo de iguales (Wilhelmsen y Sørensen, 2017). Con el objetivo de favorecer un cambio de actitudes hacia la discapacidad y mejorar la inclusión del alumnado en la etapa de Educación Primaria en el área de Educación Física, se utilizan diversas estrategias. Entre ellas podemos destacar aquellas basadas en el potencial educativo del juego, como son los juegos cooperativos y los juegos sensibilizadores.

Por un lado, los juegos cooperativos son aquellos que demandan de los jugadores una forma de actuación orientada hacia el grupo, en la que cada participante colabora con los demás para la consecución de un fin común (Omecaña y Ruiz, 2016). Estos promueven una valoración positiva de los compañeros, un incremento de relaciones sociales y facilitan la aceptación de la diversidad (Ruiz, 2008).

Por ejemplo, Lavega, Planas y Ruiz (2014) analizan las conductas motrices cooperativas de 40 niños (18 con discapacidad y 22 sin discapacidad) de 8 a 11 años, para interpretar el proceso de interacción seguido por cada uno de los alumnos participantes en un 
taller diseñado para promover la inclusión a través de juegos cooperativos. La experiencia realizada confirma que todos los participantes fueron capaces de participar activamente en los juegos de cooperación protagonizando mayoritariamente conductas motrices cooperativas ajustadas relacionadas con la empatía, el dialogo social y la socio-afectividad.

Ríos (2009) afirma que la cooperación ayuda a la participación del alumnado con discapacidad. La cooperación mejora la inclusión del alumnado con discapacidad y el exceso de competición dificulta esta inclusión. Un estudio realizado por Reina, Hutzler, IñiguezSantiago y Moreno-Murcia (2016) aborda la relación entre el nivel de habilidad y competitividad percibida, los resultados muestran que aquellos alumnos que se sentían más competitivos y habilidosos tenían una actitud hacia la discapacidad más negativa. En esta misma línea, Abellán, Sáez-Gallego y Reina (2018) muestran resultados similares en su estudio. Por lo tanto, parece que el exceso de competitividad dificulta la mejora de las actitudes hacia la discapacidad y la inclusión de alumnos con discapacidad en Educación Física, especialmente cuando la Educación Física tiene un enfoque competitivo. En este trabajo se propone orientar la Educación Física desde un enfoque cooperativo con el objetivo de favorecer la sensibilización hacia la discapacidad.

Por otro lado, los juegos sensibilizadores son aquellos que hacen que los participantes vivan de manera lúdica las limitaciones de las personas con discapacidad y valoren sus capacidades (Ríos, Blanco, Bonany y Carol, 1998). El objetivo principal de estos juegos es sensibilizar a los participantes sobre la realidad de las personas con discapacidad, fomentar actitudes positivas y de apoyo basadas en el respeto a la diversidad y evitar actitudes negativas o no integradoras a través del entretenimiento y la participación (Ríos et al., 1998).

Estos juegos han sido utilizados en estudios para concienciar, sensibilizar y cambiar la actitud de los estudiantes hacia la discapacidad y los resultados han sido positivos. Por ejemplo, Navarro (2009) analiza los efectos de unas actividades lúdicas y juegos dirigidos al alumnado de Educación Primaria con el fin de lograr una mayor y mejor integración en la dinámica escolar y una mayor sensibilización hacia los compañeros con necesidades especiales. La experiencia resulta satisfactoria, ya que se logra un grado de concienciación hacia la discapacidad. 
Experiencias didácticas Juegos cooperativos-sensibilizadores para mejorar las actitudes hacia la discapacidad en educación física en educación primaria. Vol. 8, n. ${ }^{\text {1 }}$; p. 60-80, enero 2022.

https://doi.org/10.17979/sportis.2022.8.1.8640

González y Baños (2012) analizan el cambio de actitudes hacia la discapacidad en clases de actividad física a través de la práctica de un deporte para personas con discapacidad visual (Goalball). Tras el estudio, se demuestra un cambio significativo de actitudes y creencias con respecto a compartir espacios y experiencias con alumnos con discapacidad visual. En este sentido se utiliza el deporte adaptado como un juego sensibilizador, de acuerdo a lo indicado en el trabajo de Abellán y Sáez-Gallego (2017).

Con estos antecedentes, surge el planteamiento de unir los juegos sensibilizadores y los juegos cooperativos para fomentar en las aulas de Educación Física la inclusión, evitar situaciones de rechazo o aislamiento de alumnos y mejorar las actitudes hacia la discapacidad. A continuación, se desarrolla una propuesta didáctica que ha unidos estos juegos, creando los juegos cooperativos-sensibilizadores. En esta propuesta se presenta la evaluación de la primera utilización de este tipo de juegos en el contexto de la Educación Física en Educación Primaria.

Esta experiencia didáctica se sitúa en el camino hacia la búsqueda de variables mediadoras que faciliten la sensibilización hacia la discapacidad en las clases de Educación Física. A las variables clásicas como el contacto previo o la duración de las intervenciones, se han ido añadiendo nuevas, como las diferentes estrategias didácticas empleadas en el trabajo de Reina et al. (2020) o el efecto de la competitividad analizado en el trabajo de Ocete, PérezTejero, Reina y Coterón (2020).

\section{Planteamiento didáctico}

Esta intervención une los juegos cooperativos y los juegos sensibilizadores en Educación Física en la etapa de Educación Primaria con el objetivo de mejorar las actitudes hacia la discapacidad del alumnado participante.

Los juegos cooperativos-sensibilizadores son aquellos en los cuales los participantes viven de manera lúdica las limitaciones de las personas con dificultades y valoran sus capacidades a través de juegos con objetivos comunes, que favorezcan la interrelación positiva y la colaboración entre los participantes. A continuación, se indican las principales características de estos juegos (Figura 1). 
Experiencias didácticas Juegos cooperativos-sensibilizadores para mejorar las actitudes hacia la discapacidad en educación física en educación primaria. Vol. 8, n. ${ }^{\circ}$ 1; p. 60-80, enero 2022. https://doi.org/10.17979/sportis.2022.8.1.8640

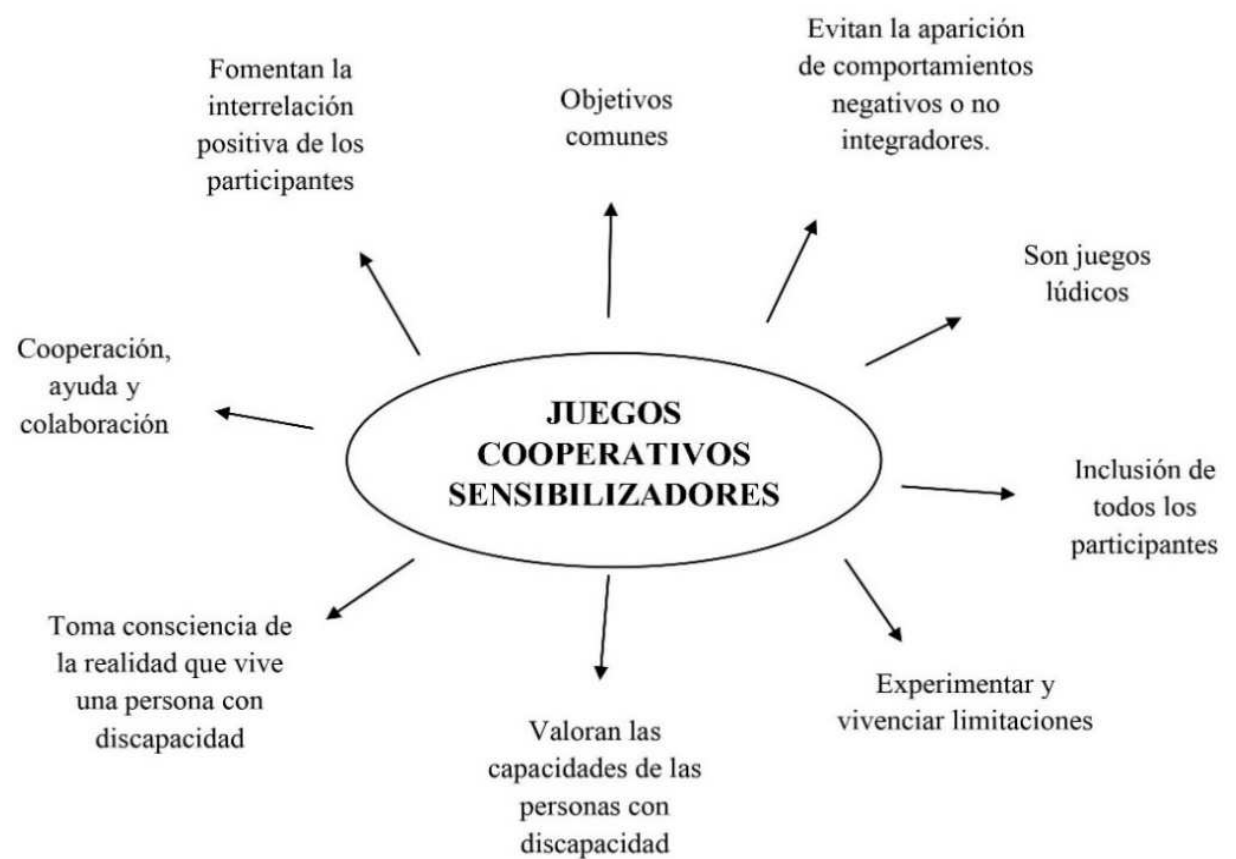

Figura 1. Principales características de los juegos cooperativos-sensibilizadores (Elaboración propia).

\section{Objetivos}

El objetivo principal de este trabajo es analizar el efecto de una intervención didáctica basada en juegos cooperativos-sensibilizadores en las actitudes hacia la discapacidad de un grupo de alumnos de quinto de primaria en el área de Educación Física.

Además, se persiguen los siguientes objetivos específicos:

- Experimentar posibles limitaciones de las personas con discapacidad desde una perspectiva lúdica.

- Valorar las capacidades de las personas con discapacidad.

- Conocer la percepción de la maestra de la intervención.

- Percibir facilitadores y barreras de la experiencia.

\section{Contexto y participantes}

El centro escolar al que se dirige la propuesta didáctica es un colegio público de una ciudad de Castilla-La Mancha (España), que tiene un cómputo general de 439 alumnos matriculados. Un total de 51 alumnos de $5^{\circ}$ curso de Educación Primaria (rango de edad $=10$ - 
Experiencias didácticas Juegos cooperativos-sensibilizadores para mejorar las actitudes hacia la discapacidad en educación física en educación primaria. Vol. 8, n. 1; p. 60-80, enero 2022.

https://doi.org/10.17979/sportis.2022.8.1.8640

11 años) de tres líneas diferentes (A, B y C) tomaron parte en la propuesta. En la Tabla 1 se indica la distribución por grupos y género.

Tabla 1. Características de los grupos participantes.

\begin{tabular}{cccc}
\hline & A & B & C \\
\hline Chicos & 10 & 9 & 7 \\
Chicas & 9 & 8 & 8 \\
Total & 19 & 17 & 15 \\
\hline
\end{tabular}

Treinta alumnos indicaron que tenían un familiar y/o amigo con discapacidad y 29 que habían tenido un compañero con discapacidad en clase. Estos alumnos nunca habían trabajado en el área de Educación Física sesiones acerca de la discapacidad, por lo que era la primera vez que participaban en este tipo de intervenciones, en este caso de juegos cooperativossensibilizadores.

La maestra de Educación Física del grupo tiene 43 años y lleva ejerciendo desde hace 17 años. La intervención fue dirigida por una maestra de Educación Primaria con Mención en Educación Física de 23 años, con 1 año de experiencia docente, durante sus prácticas en el marco de un máster profesional de Educación Física, dicha maestra es la autora principal del trabajo. Para ambas era la primera experiencia práctica con programas de sensibilización, aunque la autora principal tenía conocimientos teórico-prácticos fruto de su formación inicial tanto en el Grado como en el Máster.

\section{Desarrollo de la propuesta}

La propuesta didáctica consistió en la elaboración, desarrollo y evaluación de tres sesiones de juegos cooperativos-sensibilizadores, dicha intervención se llevó a cabo en los tres grupos participantes por separado. Cada una de estas sesiones tuvo una duración de 45 minutos y se llevaron a cabo en la misma semana.

La metodología utilizada durante las sesiones fue activa, inclusiva y participativa, promoviendo la cooperación entre los alumnos. Se creó un clima positivo, tolerante y de respeto a las diferencias. Además, se evitó el exceso de competitividad que en ocasiones surge 


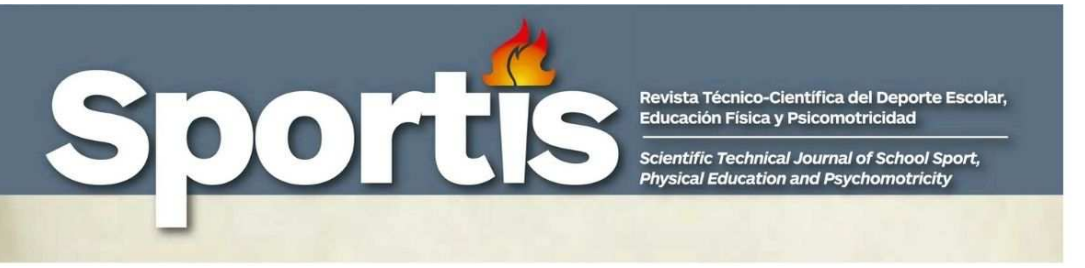

Experiencias didácticas Juegos cooperativos-sensibilizadores para mejorar las actitudes hacia la discapacidad en educación física en educación primaria. Vol. 8, n. ${ }^{\circ}$ 1; p. 60-80, enero 2022.

https://doi.org/10.17979/sportis.2022.8.1.8640

en las clases de Educación Física, las situaciones de exclusión y discriminación de alumnado, y el sentimiento de fracaso y miedo al fallo que muchos alumnos padecen en ocasiones.

Se llevaron a cabo diferentes juegos y retos cooperativos en los cuales los alumnos vivenciaban y experimentaban diversas discapacidades, ya que se utilizaba la técnica de simulación. Esta es una estrategia utilizada habitualmente en intervenciones para favorecer la inclusión en el ámbito de la Educación física, es la "simulación", fingimiento o apariencia de una discapacidad, como los cambios en el punto de vista de una persona sobre sí misma, sobre otras personas, o sobre alguna situación concreta. Sirve como recurso para alentar actitudes positivas mediante el aumento de la conciencia de la situación de las personas con discapacidad debido a que durante las simulaciones activas mejoran la autoeficacia, influyendo en las actitudes hacia la inclusión y mejorando la visión estereotipada de los compañeros con discapacidad, contribuyendo con la ruptura de falsos mitos sobre discapacidad (Ocete, Pérez-Tejero y Coterón, 2015).

En cuanto a la cooperación, entre los diferentes tipos de juegos cooperativos que existen, en esta propuesta se trabajan principalmente dos de ellos: "piensa, comparte y actúa" y "marcador/resultado colectivo". La técnica "piensa, comparte y actúa" consiste en plantear un determinado problema motor que los alumnos deben resolver como grupo. Cada alumno piensa posibles soluciones a dicho problema. Después, se exponen sus ideas a los compañeros eligen realizar una de ellas. A partir de los resultados obtenidos pueden corregir sus acciones motrices, buscando una mayor efectividad de estas, o decidir si es mejor realizar otra idea distinta (Velázquez, 2014). La técnica "marcador o resultado colectivo" consiste en realizan una determinada tarea ya sea de forma individual, en parejas o pequeños grupos colaborando hacia un objetivo común, lograr un resultado de grupo, es decir, hacer puntos de manera colectiva. Por tanto, cada alumno aporta su esfuerzo al logro global (Fernández- Río, 2017).

En la Tabla 3 se presenta el esquema de la propuesta, en la que se indica el tipo de discapacidad que se simula, el tipo de juego cooperativo y un ejemplo de juego utilizado durante la sesión. 


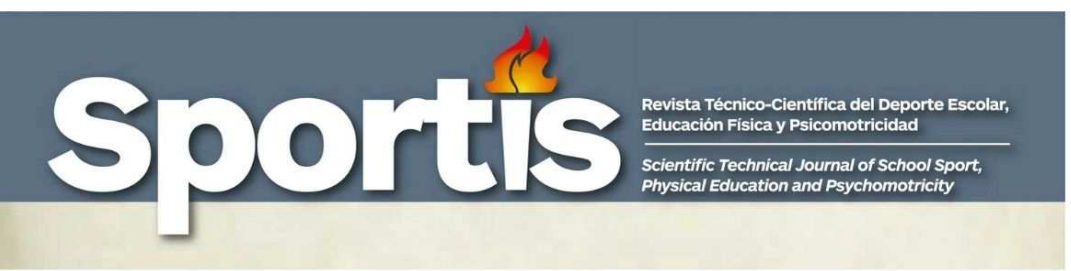

Experiencias didácticas Juegos cooperativos-sensibilizadores para mejorar las actitudes hacia la discapacidad en educación física en educación primaria. Vol. 8, n. ${ }^{\circ}$ 1; p. 60-80, enero 2022.

https://doi.org/10.17979/sportis.2022.8.1.8640

Tabla 3. Esquema de la propuesta didáctica.

\begin{tabular}{|c|c|c|c|}
\hline Sesión & Simulación & Juego cooperativo & Ejemplo \\
\hline 1 & Discapacidad visual (DV) & Marcador colectivo & $\begin{array}{l}\text { "Boom, la bomba explotó". Los } \\
\text { alumnos se agruparán en parejas, uno de ellos } \\
\text { tendrá el rol de persona con DV (se colocará } \\
\text { un pañuelo) y el otro hará de guía, } \\
\text { acompañándolo y protegiéndolo. Después } \\
\text { cambiaran de roles. Cada pareja simula que } \\
\text { es una bomba por tanto los guías deben evitar } \\
\text { que dos bombas choquen para que no } \\
\text { exploten. }\end{array}$ \\
\hline 2 & Discapacidad física (DF) & comparte, & $\begin{array}{l}\text { "Fuera de juego". Cada jugador estará } \\
\text { sentado en el suelo (simulando una DF) y } \\
\text { tendrá una pelota de goma espuma. El balón } \\
\text { de baloncesto se situará en el centro del } \\
\text { cuadrado. El objetivo es intentar sacar el } \\
\text { balón de baloncesto del cuadrado lanzando } \\
\text { las pelotas de goma espuma. }\end{array}$ \\
\hline 3 & $\begin{array}{l}\text { Discapacidad } \\
\text { (DA) }\end{array}$ & comparte, & $\begin{array}{l}\text { “Cómo pasamos el río?”. Se } \\
\text { dispondrán todos los grupos inicialmente en } \\
\text { un lado del río con los oídos tapados } \\
\text { (simulando una DA). El objetivo es formar } \\
\text { un puente con sus materiales para que todos } \\
\text { los miembros del grupo puedan cruzar el río a } \\
\text { través de la interacción y la comunicación no } \\
\text { verbal. }\end{array}$ \\
\hline
\end{tabular}

\section{Evaluación}

Se realizó una evaluación con dos momentos de medida, pre-test y post-test, para conocer si se habían conseguido los objetivos planteados tras la implementación de la propuesta didáctica de juegos cooperativos-sensibilizadores. Para ello se utilizaron los siguientes instrumentos: 

educación física en educación primaria. Vol. 8, n. ${ }^{\text {1 }}$; p. 60-80, enero 2022.

https://doi.org/10.17979/sportis.2022.8.1.8640

- Cuestionario sociodemográfico: se analizaron variables vinculadas al: i) género (chico o chica); ii) haber tenido contacto con familiares o amigos con discapacidad (" ¿Tienes un familiar o amigo/a cercano con discapacidad?’); y iii) contacto con compañeros con discapacidad en clases de EF (“¿Has tenido alguna vez un compañerola con discapacidad en tu clase de educación física?"). Para informar acerca del género, los estudiantes marcaban la opción correspondiente, y para el resto de las preguntas había que elegir entre las opciones: "S $\imath$ " o "No".

- Escala de Actitud hacia el Alumnado con Discapacidad en Educación Física (EAADEF) de Íñiguez-Santiago, Ferriz, Martínez-Galindo, Cebrián-Sánchez y Reina, (2017). La escala original estaba destinada a alumnado de Educación Secundaria. Ha sido recientemente validada para alumnado de Educación Primaria por Abellán, Ferriz, Sáez-Gallego y Reina (2020).

Este instrumento comienza con la frase introductoria "En Educación Física, con respecto a las personas con discapacidad...”. Esta escala incluye cuatro ítems, los cuales están relacionados con el componente conductual de la actitud (por ejemplo: “no propondría como capitán de mi equipo a una persona con discapacidad”). Las respuestas se recogen por medio de una escala tipo Likert de 1 (totalmente en desacuerdo) a 5 (totalmente de acuerdo). Las puntuaciones más altas en esta escala revelan una actitud menos positiva hacia la participación del alumnado con discapacidad en el área de Educación Física. Se incluye además una primera pregunta de control ("Voy al colegio en [ciudad en la que se realizó la intervención]”) para garantizar que los alumnos comprendían el procedimiento a seguir para completar el instrumento.

Siguiendo las directrices de Íñiguez-Santiago et al. (2017), se añadió al instrumento la explicación del concepto de discapacidad: "una persona con discapacidad es aquella, que debido a la alteración de una estructura o función corporal (física, sensorial o intelectual), tiene limitaciones a la hora de realizar las actividades que serían normales". Antes de que el alumnado respondiera el cuestionario, la maestra explicó el concepto de discapacidad, incluyendo ejemplos y resolviendo las dudas que pudieran surgir. Este instrumento se administró a los participantes tanto antes como 


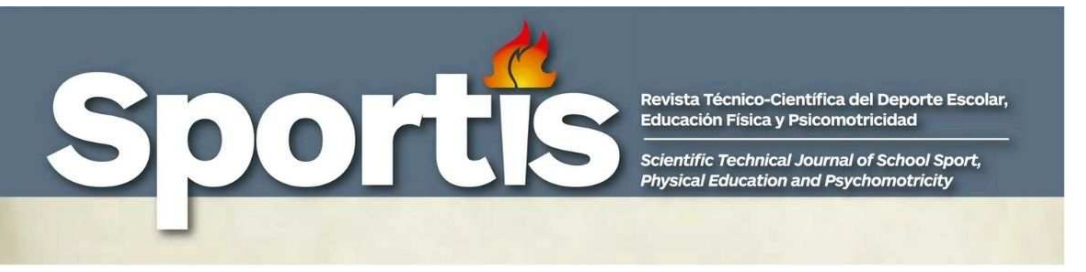

Experiencias didácticas Juegos cooperativos-sensibilizadores para mejorar las actitudes hacia la discapacidad en educación física en educación primaria. Vol. 8, n. ${ }^{\circ}$ 1; p. 60-80, enero 2022.

https://doi.org/10.17979/sportis.2022.8.1.8640

después de la intervención de juegos cooperativos-sensibilizadores, lo que sirvió como pre-test y post-test. El cuestionario ha mostrado una alta fiabilidad en la población del presente trabajo $(\alpha=, 879)$.

- Grupo de discusión: Se invitó a participar a nueve alumnos, tres de cada grupo. Como criterios de participación se establecieron los siguientes: haber estado presente en las tres sesiones de juegos cooperativos-sensibilizadores, mostrar una actitud participativa y capacidad para expresar sus opiniones respecto a la intervención. Para la selección de la muestra se solicitó la ayuda de la maestra de Educación Física. El objetivo era conocer las ideas de los participantes acerca de la discapacidad, sus experiencias en los juegos realizados, cómo se habían sentido y si había sido importante la cooperación y la ayuda. Para ello, en primer lugar, se realizaron preguntas sobre las personas con discapacidad y posteriormente sobre sus experiencias en los juegos cooperativos-sensibilizadores. Algunos ejemplos de preguntas: “Creéis que las personas con discapacidad tienen problemas o dificultades en su día a día?”, “¿Pensáis que necesitan nuestra ayuda?”, “CCómo os sentisteis en los juegos?”, “Os ayudabais unos a otros para conseguir el objetivo de cada juego?”, “Creéis que es importante ayudarse unos a otros?”.

- Entrevista semiestructurada: Se realizó a la maestra de Educación Física una entrevista con el objetivo de conocer su opinión en cuanto a las sesiones realizadas de juegos cooperativos-sensibilizadores. Algunas preguntas realizadas fueron: “Crees que ha sido positivo llevar a cabo esta intervención?”, “Qué han aprendido los alumnos?”, “¿Piensas que es importante trabajar estos contenidos en Educación Física?”.

Se obtuvieron los consentimientos informados para participar de los tutores legales del alumnado participante, así como de la maestra y de los responsables del centro. La administración de las pruebas se llevó a cabo en el aula de cada grupo, durante el horario lectivo y con la presencia de la maestra de Educación Física. Se garantizó el anonimato de todos los participantes. Durante el proceso de toma de datos se respetaron las recomendaciones éticas de la American Psychological Association (2010). 
Experiencias didácticas Juegos cooperativos-sensibilizadores para mejorar las actitudes hacia la discapacidad en educación física en educación primaria. Vol. 8, n. ${ }^{\circ}$ 1; p. 60-80, enero 2022. https://doi.org/10.17979/sportis.2022.8.1.8640

\section{Análisis de los datos}

Para los datos cuantitativos se ha utilizado el programa Excel para recopilar todos los datos de los participantes. Posteriormente se ha utilizado el SPSS.23 para analizar estos datos. En primer lugar, se llevó a cabo la prueba de normalidad y una vez comprobado que la distribución de los datos no cumplía con los criterios de normalidad, se realizaron pruebas no paramétricas, en concreto, para comparar el efecto de la intervención en las actitudes hacia la discapacidad en Educación Física se realizó la comparación por pares (pre-test, post-test) utilizando la prueba de los rangos con signo de Wilcoxon.

El análisis de los datos cualitativos recogidos se hizo a través de una identificación de las unidades de análisis, la posterior agrupación de estás en dos categorías (facilitadores y barreras) y los códigos asignados a estas dos categorías, dentro de facilitadores: experiencias previas, ayuda, cooperación, valoración de capacidades, confianza, empatía y concienciación y dentro de barreras, limitaciones y miedo. Para ello se ha utilizado el programa Atlas.ti. Se realizó un análisis inicial deductivo para las categorías y tras él, un análisis posterior inductivo para la generación de códigos. La codificación inicial fue realizada por la autora principal del trabajo. Los códigos resultantes fueron ampliamente discutidos con el segundo autor, que ejerció el papel de amigo crítico. Fruto de este proceso surgió la codificación final que se presenta en el apartado siguiente.

\section{Resultados}

\section{Resultados cuantitativos}

Los resultados de las actitudes hacia la inclusión en Educación Física se presentan en la Tabla 4. 
Experiencias didácticas Juegos cooperativos-sensibilizadores para mejorar las actitudes hacia la discapacidad en educación física en educación primaria. Vol. 8, n. ${ }^{\circ}$ 1; p. 60-80, enero 2022.

https://doi.org/10.17979/sportis.2022.8.1.8640

Tabla 4. Resultados de las actitudes hacia la inclusión en Educación Física (M+DT).

\begin{tabular}{llc}
\hline & Pre-test & Post-test \\
\hline Prefiero no relacionarme con personas con & $1,79 \pm 1,33$ & $1,35 \pm 0,80$ \\
discapacidad* & & $1,49 \pm 0,80$ \\
Evitaría hacer un trabajo de clase con una & $1,76 \pm 1,25$ & $1,42 \pm 0,83$ \\
persona con discapacidad. & $1,89 \pm 1,32$ & $1,53 \pm 1,08$ \\
$\begin{array}{l}\text { Evitaría para mi equipo a una persona con } \\
\text { discapacidad* }\end{array}$ & $1,84 \pm 1,34$ & $1,44 \pm 0,69$ \\
No propondría como capitán de mi equipo a & & \\
una persona con discapacidad. & $1,82 \pm 1,20$ & \\
\hline EAADEF Total & & \\
\hline
\end{tabular}

Nota. Asterisco (*) indica diferencias significativas para $p<0,05$.

La prueba de Wilcoxon no ha arrojado diferencias significativas para $\mathrm{p}<0,05$ para el total de la escala. Al realizar un análisis ítem a ítem, la prueba de Wilcoxon revela diferencias significativas en el ítem $1(Z=-2,27, p=, 023)$ y en el ítem $3(Z=-2,39, p=, 017)$. Después de la intervención, los participantes puntuaron significativamente más bajo en estos ítems, mostrando una actitud más positiva hacia la discapacidad en Educación Física.

\section{Resultados cualitativos}

Los resultados obtenidos recogen un total de 68 citas distribuidas en 9 códigos que conforman dos categorías principales: facilitadores y barreras, tal como se puede observar en la Tabla 5. 


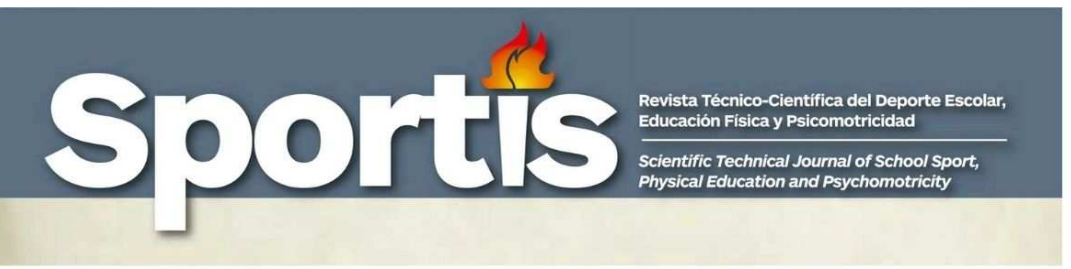

Experiencias didácticas Juegos cooperativos-sensibilizadores para mejorar las actitudes hacia la discapacidad en educación física en educación primaria. Vol. 8, n. ${ }^{\circ}$; p. 60-80, enero 2022.

https://doi.org/10.17979/sportis.2022.8.1.8640

Tabla 5. Clasificación de códigos y citas en sus respectivas categorías.

\begin{tabular}{llc}
\hline Categoría & Códigos & Citas \\
\hline & Ayuda & 7 \\
Facilitadores & Cooperación & 4 \\
& Valoración de capacidades & 12 \\
& Confianza & 6 \\
& Empatía & 11 \\
& Concienciación & 10 \\
\hline Barreras & Limitaciones & 9
\end{tabular}

La categoría de facilitadores es la más representativa a través de 6 códigos: ayuda, cooperación, valoración de capacidades, confianza, empatía y concienciación (un total de 53 citas). Los nombres que se indican corresponden a Seudónimos de los participantes.

Los participantes comentan sus experiencias vividas durante la intervención de juegos cooperativos-sensibilizadores. En cuanto a cooperar y ayudarse unos a otros surgen los siguientes comentarios: "en el juego del puzle teníamos que ayudarnos a guiarnos porque como había tantos obstáculos a lo mejor si no le guiabas bien no se podía hacer” indica Hugo, y Emma continuaba diciendo "y también en el juego de las películas había que cooperar todos para hacerlo bien y los demás entendiesen que película era”. La maestra lo confirma indicando "se han ayudado y han cooperado entre todos durante los diferentes juegos en todo momento”.

Posteriormente comentan cómo se sentían. "A ver... en algunos me sentía rara porque nunca había ido sin mirar o sin oír o algo, y me sentía rara pero luego ya normal” decía Inés, "Yo sentí... no compasión, pero no sé qué... las personas con discapacidad tienen que hacer un gran esfuerzo, igual que nosotros, que nosotros estuvimos 5 minutos y ya estábamos que no podíamos más” dice Candela e Inés reafirma esta contestación y añade "y también emoción”.

Se pregunta a los alumnos qué aprendieron y las respuestas fueron las siguientes. Emma: "pues a ver, a mí me ha ayudado a entender a las personas con discapacidad...", 
Experiencias didácticas Juegos cooperativos-sensibilizadores para mejorar las actitudes hacia la discapacidad en educación física en educación primaria. Vol. 8, n. ${ }^{\circ}$ 1; p. 60-80, enero 2022.

https://doi.org/10.17979/sportis.2022.8.1.8640

Inés responde "es verdad no es tan fácil como la gente cree, tienes que confiar en otra persona y es muy difícil”, Candela argumenta:

"Al hacerlo y al sentir que de verdad esa persona o no ve o lo que sea y ya te pones en su piel pues ya te das cuenta de que a lo mejor no hace falta reírse de él porque para esa persona le puedes ofender y para él realmente es complicado".

La entrevistadora para finalizar pregunta " ¿y creéis que es importante trabajar esto en el colegio como hemos hecho ahora?, Hugo responde "si, para concienciarse" e Inés "para que la gente vea que no es tan fácil y que si tu estuvieses ahí no te gustaría que se rieran de ti, que no te ayudasen...". La maestra añade "ha sido una intervención muy positiva...los alumnos han experimentado diversas discapacidades, se han puesto en la piel de personas con discapacidad y han aprendido mucho... creo que es muy importante trabajar este aspecto en esta área...”.

Sin embargo, también observamos diversas barreras en la puesta en práctica de esta experiencia docente. Esta categoría, compuesta por 2 códigos (15 citas en total), implica limitaciones y miedo. Respecto a las limitaciones, los alumnos muestran diferentes tipos de limitaciones ante los juegos cooperativos-sensibilizadores y las relacionan con las que pueden llegar a tener las personas con discapacidad. "No es tan fácil para personas con discapacidad hacer algunas actividades" argumenta Nadia, Silvia añade "su cuerpo no está preparado y no pueden hacer algunas cosas...”.

En cuanto al miedo Candela comenta "en el que había que taparse los ojos, no sé, tenía como miedo porque al ir con los ojos tapados y no estar acostumbrada pues sentía que me iba a chocar", algo similar dice Marcos "daba miedo por si te chocabas, me sentía incómodo" e Inés añade "yo es que como que no terminé de confiar".

\section{Discusión}

El objetivo del presente trabajo era analizar el efecto de una intervención de juegos cooperativos-sensibilizadores, llevada a cabo con el alumnado de quinto de Educación Primaria, sobre las actitudes hacia la discapacidad en Educación Física.

Se presenta una perspectiva novedosa debido a que se ha elaborado, desarrollado y evaluado una intervención en la que se han unido los juegos cooperativos y los juegos 

educación física en educación primaria. Vol. 8, n. ${ }^{\text {1 }}$; p. 60-80, enero 2022.

https://doi.org/10.17979/sportis.2022.8.1.8640

sensibilizadores. De este modo, este trabajo supone la primera aplicación práctica de esta propuesta de juegos cooperativos-sensibilizadores en Educación Física en la etapa de Educación Primaria, obteniendo resultados positivos, la mejora de las actitudes hacia la discapacidad y el fomento de valores como el respeto a las diferencias y la inclusión.

La práctica de una serie de juegos cooperativos-sensibilizadores ha conseguido que los participantes mejoraran sus actitudes hacia la discapacidad en Educación Física en todos los ítems del cuestionario EAADEF, aunque solamente en dos de ellos dichas mejoras han sido significativas. Estos resultados indican que el alumnado obtiene mejores valores tras la intervención, al igual que muestran por ejemplo Hutzler, Fliess-Douer, Avraham, Reiter y Talmor (2007), McKay, Block y Park (2015) o Pérez-Torralba, Reina, Pastor-Vicedo y González-Víllora, (2018).

Por ejemplo, Hutzler et al. (2007) llevaron a cabo un estudio con el objetivo de explorar el efecto de dos actividades de concienciación sobre las actitudes de los niños hacia sus compañeros con discapacidad. En la primera actividad participaron activamente en un taller simulando actividades de movimiento, mientras que en la segunda intervención observaron a sus compañeros participando en una actividad de baloncesto en silla de ruedas junto con jugadores de élite de baloncesto en silla de ruedas. Los resultados indicaron efectos positivos significativos en los dominios cognitivos y conductual de las actitudes hacia la discapacidad.

Pérez-Torralba et al., (2018) llevaron a cabo una investigación que utilizaba los paradeportes, en concreto la Boccia y el Goalball, para atletas con altas necesidades de apoyo para sensibilizar a los estudiantes hacia la discapacidad en sus clases de Educación Física con participantes de sexto curso de Primaria. Los resultados revelaron actitudes positivas hacia la discapacidad en los alumnos que realizaron la intervención.

Por otra parte, existen evidencias científicas que muestran que los juegos cooperativos favorecen la inclusión del alumnado. Por ejemplo, Lavega et al. (2014) analizaron las conductas motrices cooperativas de 40 niños (18 con discapacidad y 22 sin discapacidad) de 8 a 11 años, para interpretar el proceso de interacción seguido por cada uno de los alumnos participantes en un taller diseñado para promover la inclusión a través de juegos cooperativos. La experiencia realizada confirmó que todos los participantes fueron capaces de participar 
Experiencias didácticas Juegos cooperativos-sensibilizadores para mejorar las actitudes hacia la discapacidad en educación física en educación primaria. Vol. 8, n. ${ }^{\circ}$ 1; p. 60-80, enero 2022.

https://doi.org/10.17979/sportis.2022.8.1.8640

activamente en los juegos de cooperación protagonizando mayoritariamente conductas motrices cooperativas ajustadas relacionadas con la empatía, el dialogo social y la socioafectividad.

Además, se recalca que este cambio de actitudes ha sido posible debido a diversos facilitadores como son las actitudes y valores positivos por parte de los participantes y de la maestra como se observa en los resultados obtenidos en el estudio cualitativo. Como menciona Martínez-Figueira (2013) se deben tener en cuenta los indicadores que permiten reconocer buenas prácticas inclusivas, señalando las actitudes y valores por parte de toda la comunidad educativa, definidos como la integración de todos, la aceptación de las diferencias y la normalidad que esto lleva consigo, la igualdad de oportunidades y acceso, la sensibilización hacia el cambio, el respeto y la tolerancia. También destacan la participación y colaboración.

Sin embargo, se ha podido observar una barrera, el miedo. Los alumnos al ponerse en la piel de una persona con discapacidad han experimentado ciertos sentimientos que han dificultado su manera de desenvolverse en las diferentes situaciones dadas durante los juegos por el miedo a lo que pudiera ocurrir más allá de lo que estaba a su alcance. Martos-García y Valencia-Peris (2016) afirman que el miedo y la desconfianza provocado por el desconocimiento son la peor barrera. También Cordente-Mesas, González-Víllora, Block y Contreras-Jordan (2016) señalan que es habitual que los alumnos presenten miedo a la hora de experimentar una discapacidad en primera persona, por ejemplo, miedo a lo desconocido, a caerse, desorientarse o a no confiar en su guía, especialmente en el caso de la simulación de la discapacidad visual. Por tanto, ofrecer un clima de seguridad y confianza es primordial para disminuir estos miedos causados.

\section{Conclusiones}

La aplicación del programa de intervención basado en la aplicación de juegos cooperativos-sensibilizadores ha sido una experiencia positiva, que ha conseguido unir los juegos cooperativos y los juegos sensibilizadores consiguiendo mejorar las actitudes hacia la discapacidad en Educación Física de los estudiantes de quinto de Educación Primaria. 
Experiencias didácticas Juegos cooperativos-sensibilizadores para mejorar las actitudes hacia la discapacidad en educación física en educación primaria. Vol. 8, n. ${ }^{\circ}$ 1; p. 60-80, enero 2022.

https://doi.org/10.17979/sportis.2022.8.1.8640

La alta motivación del alumnado, su interés y participación han ayudado a lograr que se pudiera desarrollar la intervención correctamente, logrando la sensibilización de los alumnos hacia la sensibilidad, la inclusión y el cambio de actitudes.

Se remarca la necesidad de incluir juegos cooperativos-sensibilizadores en las programaciones de Educación Física en Primaria, para que de esta forma el alumnado pueda conocerlos y experimentar en primera persona las sensaciones que presentan las personas con algún tipo de discapacidad, fomentando valores como el respeto a las diferencias y la inclusión.

Finalmente, hay que destacar que se han detectado una serie de limitaciones como la falta de un grupo control y la escasez de la muestra. Un grupo control hubiera permitido comparar los resultados de manera confiable con el grupo experimental. Pudiéndose observar otras variables que pudieran influir en los resultados obtenidos. Además, la escasez de la muestra impide extrapolar estas conclusiones al resto de la población de alumnos de Educación Primaria. Se establece la necesidad de seguir investigando en esta línea para poder determinar la efectividad de este tipo de programas sobre las actitudes hacia las personas con discapacidad en las clases de Educación Física en Primaria. De manera que se propone como línea de investigación futura la valoración del efecto de la práctica de juegos cooperativos sensibilizadores como contenido de Educación Física en la etapa de Educación Primaria.

\section{Referencias bibliográficas}

Abellán, J., Ferriz, R., Sáez-Gallego, N. M., y Reina, R. (2020). Actitudes hacia el alumnado con discapacidad en educación física: Validación de la EAADEF-EP a la etapa de educación primaria. Cultura, Ciencia y Deporte, 15(44), 235-243. https://doi.org/10.12800/ccd.v15i44.1465

Abellán, J., Sáez-Gallego, N. M., y Reina, R. (2018). Evaluación de las actitudes hacia la discapacidad en educación física: Efecto diferencial del sexo, contacto previo y la percepción de habilidad y competencia. Cuadernos de Psicología del Deporte, 18(1), 133-140. 
Experiencias didácticas Juegos cooperativos-sensibilizadores para mejorar las actitudes hacia la discapacidad en educación física en educación primaria. Vol. 8, n. 1; p. 60-80, enero 2022.

https://doi.org/10.17979/sportis.2022.8.1.8640

Abellán, J., y Sáez-Gallego, N. (2017). Los para-deportes como contenido de educación física en primaria: El ejemplo de la boccia. Didacticae: Revista de Investigación en Didácticas Específicas, 2, 134-142. https://doi.org/10.1344/did.2017.2.134-142

American Psychological Association (2010). Publication Manual of the American Psychological Association (6 ${ }^{\mathbf{a}}$ ed.). Washington, DC: American Psychological Association.

Cordente-Mesas, D., González-Víllora, S., Block, M. E., y Contreras-Jordán, O. R. (2016). Structure, validity and reliability of the Children's Attitudes Towards Integrated Physical Education-Spanish version (CAIPE-SP). European Journal of Adapted Physical Activity, 9(2). https://doi.org/10.5507/euj.2016.005

Fernández-Río, J. (2017). El Ciclo del Aprendizaje Cooperativo: una guía para implementar de manera efectiva el aprendizaje cooperativo en educación física. Retos ,32, 264-269. https://doi.org/10.47197/retos.v0i32.51298

González, J. y Baños, L.M. (2012). Estudio sobre el cambio de actitudes hacia la discapacidad en clases de actividad física. Cuadernos de Psicología del deporte, 12 (2), 101-108. https://doi.org/10.4321/S1578-84232012000200011

Hutzler, Y., Fliess-Douer, O., Avraham, A., Reiter, S., y Talmor, R. (2007). Effects of shortterm awareness interventions on children's attitudes toward peers with a disability. International Journal of Rehabilitation Research, 30(2), 159-161. https://doi.org/10.1097/mrr.0b013e32813a2eb6

Íñiguez-Santiago, M. C., Ferriz, R., Martínez-Galindo, M. C., Cebrián- Sánchez, M. M., y Reina, R. (2017). Análisis factorial de la escala de actitudes hacia el alumnado con discapacidad en educación física (EAADEF). Psychology, Society, \& Education, 9(3), 493-504. https://doi.org/10.25115/psye.v9i3.652

Lavega, P., Planas, A. y Ruiz, P. (2014). Juegos cooperativos e inclusión en educación física. Revista International de Medicina y Ciencias de la Actividad Física y del Deporte, 53(14), 37-51.

Martínez-Figueira, E. (2013). Una mirada hacia la inclusión: barreras en el camino a la participación. Perspectiva Educacional. Formación de Profesores, 52 (2), 177-200. https://doi.org/10.4151/07189729-vol.52-iss.2-art.101 
Experiencias didácticas Juegos cooperativos-sensibilizadores para mejorar las actitudes hacia la discapacidad en educación física en educación primaria. Vol. 8, n. 1; p. 60-80, enero 2022.

https://doi.org/10.17979/sportis.2022.8.1.8640

Martos-García, D. y Valencia-Peris, A. (2016). Osteogénesis imperfecta y educación física: Un caso inédito de inclusión educativa. Estudios pedagógicos (Valdivia), 42(1), 159175. https://doi.org/10.4067/s0718-07052016000100010

McKay, C., Block, M. y Park, J. Y. (2015). The impact of paralympic school day on student attitudes toward inclusion in physical education. Adapted Physical Activity Quarterly, 32(4), 331-348. https://doi.org/10.1123/apaq.2015-0045

Ministerio de Educación y Formación Profesional - Gobierno de España (2021). Estadística de las enseñanzas no universitarias. Alumnado con necesidad específica de apoyo educativo curso 2019-2020.

Extraído de https://www.educacionyfp.gob.es/dam/jcr:a3a6c703-ba61-4027-8ee4bbb0aa2e46ce/notaresumen20.pdf

Navarro, E. (2009). "Juegos sensibilizadores" Los juegos motrices sensibilizadores: un medio hacia la normalización y el respeto a la diversidad. Revista digital innovación y experiencias educativas. 14, 1-8.

Ocete, C., Pérez-Tejero, J. y Coterón, J. (2015). Propuesta de un programa de intervención educativa para facilitar la inclusión de alumnos con discapacidad en educación física. Retos. Nuevas Tendencias en Educación Física, Deporte y Recreación, (27),1 40-145. https://doi.org/10.47197/retos.v0i27.34366

Ocete, C., Pérez-Tejero, J., Coterón, J. y Reina, R. (2020). How do competitiveness and previous contact with people with disabilities impact on attitudes after an awareness intervention in physical education? Physical Education and Sport Pedagogy, 1-13. https://doi.org/10.1080/17408989.2020.1834527

Omecaña, R y Ruiz, J.V. (2016). Juegos cooperativos y educación física. Barcelona: Paidotribo.

Pérez-Torralba, A., Reina, R., Pastor-Vicedo, J.C y González-Víllora, S. (2018): Education intervention using para-sports for athletes with high support needs to improve attitudes towards students with disabilities in Physical Education. European Journal of Special Needs Education, 34(4), 455-468. https://doi.org/10.1080/08856257.2018.1542226

Reina, R., Hutzler, Y., Iñiguez-Santiago, M. C., y Moreno-Murcia, J. A. (2016). Attitudes towards inclusion of students with disabilities in Physical Education Questionnaire 
Experiencias didácticas Juegos cooperativos-sensibilizadores para mejorar las actitudes hacia la discapacidad en educación física en educación primaria. Vol. 8, n. ${ }^{\circ}$ 1; p. 60-80, enero 2022.

https://doi.org/10.17979/sportis.2022.8.1.8640

(AISDPE): A two-component scale in Spanish. European Journal of Human Movement, 36, 75-87.

Reina, R., Íñiguez-Santiago, M. C., Ferriz-Morell, R., Martínez-Galindo, C., CebriánSánchez, M. y Roldan, A. (2020). The effects of modifying contact, duration, and teaching strategies in awareness interventions on attitudes towards inclusion in physical education. European Journal of Special Needs Education, 1-17. https://doi.org/10.1080/08856257.2020.1842973

Ríos, M. (2009). La Inclusión en el Área de Educación Física en España. Análisis de las Barreras para la Participación y Aprendizaje. Ágora para la EF y el Deporte, 9, 83114.

Ríos, M., Blanco, A., Bonany, T. y Carol, N. (1998). El juego y los alumnos con discapacidad. Madrid: Paidotribo.

Ruiz, J. V. (2008). El juego motor cooperativo, ¿un buen contexto para la enseñanza?... Cuando la Educación Física nos hace más humanos. Educación Física y deporte, 27(1), 97-112. https://doi.org/10.47197/retos.v0i9.35052

Simoni, C., Santillana, H. y Yáñez, A. (2013) La inclusión y el aprendizaje cooperativo en la sesión de educación física a través del Puzzle de Aronson. "La Peonza"- Revista de Educación Física para la paz, 8. https://doi.org/10.35376/10324/2823

Velázquez, C. (2014). Aprendizaje cooperativo: aproximación teórico-práctica aplicada a la educación física. EmásF: Revista Digital de Educación Física, 29, 19-31.

Wilhelmsen, T., y Sørensen, M (2017). Inclusion of children with disabilities in physical education: A systematic review of literature from 2009 to 2015. Adapted Physical Activity Quarterly, 34(3), 311-337. https://doi.org/10.1123/apaq.2016-0017 